\title{
Synthesis of silver nanoparticles by using Aloe vera and Thuja orientalis leaves extract and their biological activity: a comprehensive review
}

\author{
Prashant J. Burange ${ }^{1 *}\left(\mathbb{C}\right.$, Mukund G. Tawar ${ }^{2}$, Ritu A. Bairagi ${ }^{1}$, Vedanshu R. Malviya ${ }^{2}$, Vanshika K. Sahu', \\ Sakshi N. Shewatkar ${ }^{1}$, Roshani A. Sawarkar ${ }^{1}$ and Renuka R. Mamurkar ${ }^{1}$
}

\begin{abstract}
Background: Nanotechnology's rapid development has been in great demand, particularly for silver nanoparticles, which are useful in a variety of industries including medicine, textiles, and home appliances. Silver nanoparticles are extremely essential due to their unique physicochemical and antibacterial properties, which can be used in a variety of applications. Green synthesis is an environmentally friendly alternative to conventional synthesis because it uses fewer chemical reagents and lowers temperature and pressure. Aloe vera and Thuja orientalis have a wide medical use because it contains a large number of compounds derived and was decided to use for the synthesis of nanoparticles.

Main text: The combination of silver nanoparticles has a wide range of applications, which has encouraged researchers to focus on the methods for the synthesis of silver nanoparticles from Aloe vera and Thuja orientalis leaves extract, characterization techniques of synthesized silver nanoparticles, and evaluation of their antimicrobial and antifungal activities. The synthesized AgNPs can be characterized by using various analytical techniques including UV-visible spectroscopy, Fourier transform infrared spectroscopy (FTIR), scanning electron microscopy (SEM), etc. The various types of silver nanoparticles, various strategies for silver nanoparticle synthesis, nano-based drug delivery systems, taxonomy and chemical constituents of Aloe vera and Thuja orientalis plants, the general mechanism of action of silver nanoparticles in bacteria, and various applications of silver nanoparticles have also been discussed.

Conclusion: This review covers a wide range of research on silver nanoparticles to gain a better understanding of their physicochemical feature characterization, production, mechanisms of action, and applications. Various AgNP factors, such as size, surfactant, and structural shape, influence the unique physicochemical properties of these nanoparticles. Even though there are a variety of ways to make AgNPs, green synthesis has a high yield and biocompatibility because it uses natural agents and harmless chemicals. In this paper, we describe the green manufacture of silver nanoparticles utilizing Aloe vera and Thuja orientalis leaf extracts, as well as the method to test their antimicrobial and antifungal activity.
\end{abstract}

Keywords: Silver nanoparticles, Green synthesis, Aloe, Thuja

\footnotetext{
*Correspondence: prashantburange@gmail.com

1 Department of Pharmaceutical Chemistry, P. R. Pote Patil College

of Pharmacy, Amravati 444602, India

Full list of author information is available at the end of the article
}

\section{Background}

Nanotechnology is the science of manipulating and using matter on a microscopic scale. It allows for the production of new materials, particularly those for medical uses, where older methods may be restrictive. 
Nanotechnology should not be regarded as a single approach with limited application (Galatage et al. 2020). Because of their unique properties and the vast variety of applications, silver nanoparticles (AgNPs) have been widely studied for decades. The use of microorganisms or plant extracts to synthesize AgNPs has emerged as a viable alternative. There are several advantages to using biosynthetic techniques. They are easy to use, cost-effective, provide great yields, and are eco-friendly (Tippayawat et al. 2016). The majority of chemical approaches for synthetic nanoparticles are prohibitively expensive and involve the use of toxic, dangerous compounds that pose a variety of biological concerns. This increases the need for ecologically friendly processes to be developed using green synthesis and other biological approaches. The production of nanoparticles utilizing diverse plants and their extracts might sometimes be more advantageous than other biological synthetic processes, which need extremely complex microbial culture maintenance procedures. The physicochemical properties of AgNPs are influenced by the size, morphology, surface, and particle distribution of nanoparticles, which can be changed using various synthetic processes, reducing agents, and stabilizers (Salleh et al. 2020). Size has been the determining factor of the biological properties of AgNPs and can be adjusted according to the specific application, typically in the range of $2-100 \mathrm{~nm}$. For example, the size of AgNPs for drug delivery applications must be greater than $100 \mathrm{~nm}$ to accommodate the number of drugs to be delivered (Patra et al. 2015). The biological activity of AgNPs is regulated by the size distribution, surface chemistry, particle shape, chemical composition, agglomeration, capping agent, particle response in media, ion release, and the reducing agents utilized during AgNP synthesis (Prakash, et al. 2017). Nanosilver has been shown to have antibacterial, fungicidal, and wound-healing effects. The low level of silver ion release from the nanoparticle surface is principally responsible for these effects. According to extensive studies, silver nanoparticles are also less harmful than silver salts of equivalent mass (Gajbhiye and Sakharwade 2016). Fungal infections are more common in immunocompromised patients, and conquering fungimediated disorders is a time-consuming procedure due to the limited number of antifungal medications available at the moment. As a result, developing antifungal agents that are biocompatible, non-toxic, and environmental friendly is a foreseeable and pressing demand. AgNPs performed a significant function as anti-fungal agents against many fungal-caused diseases to help solve the problem (Verma and Maheshwari 2019).
Here is an overview of nanoparticles, their types, and their applications in various domains, with a focus on the green synthesis of silver nanoparticles from Aloe vera and Thuja orientalis leaf extract and their antifungal efficacy.

\section{Types of nanoparticles}

The nanoparticles are generally classified into organic, inorganic, and carbon-based (Sharma et al. 2019). Figure 1 includes the types of nanoparticles.

\section{Organic nanoparticles}

Dendrimers, micelles, liposomes, ferritin, etc., are all kinds of organic nanoparticles. Biodegradable and nontoxic nanoparticles like micelles and liposomes have a hollow core and are sensitive to heat and electromagnetic waves. As a consequence of their special properties, they are a perfect alternative for drug delivery. Entrapped medication or adsorbable medication systems verify their field of application and their potency except for their traditional characteristics, like size, composition, surface morphology, and alternative such traditional characteristics. Drug delivery systems involving organic nanoparticles are widely utilized in the medical field as a result of which they operate well and might be injected into specific components of the body; this can be called targeted drug delivery (Sharma et al. 2019).

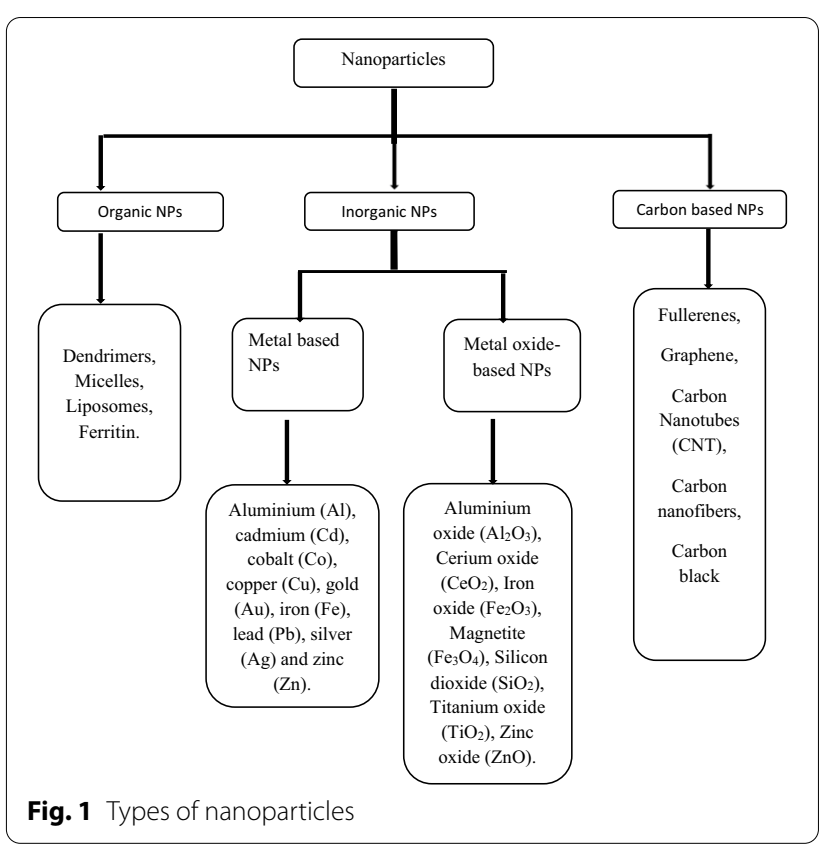




\section{Inorganic nanoparticles}

Inorganic nanoparticles are particles that are not made from carbon. Metal and metal oxide-based nanoparticles are generally categorized as inorganic nanoparticles (Sharma et al. 2019).

\section{a) Metal-based nanoparticles}

Metal-based nanoparticles are made by reducing metals to nanometric sizes using destructive or constructive methods. Metal nanoparticles are produced from the majority of metals. The unremarkably used metals for nanoparticle synthesis are aluminum ( $\mathrm{Al})$, cadmium $(\mathrm{Cd})$, cobalt $(\mathrm{Co})$, copper $(\mathrm{Cu})$, gold $(\mathrm{Au})$, iron $(\mathrm{Fe})$, lead $(\mathrm{Pb})$, silver $(\mathrm{Ag})$, and zinc $(\mathrm{Zn})$. Surface characteristics like high surface-area-to-volume magnitude relation, pore size, and surface charge density differentiate nanoparticles from alternative materials. Crystalline and amorphous structures distinguish nanoparticles from alternative materials. For starters, gold and silver nanoparticles, which have superior material properties and practical skills, are gaining the right smart attention from researchers. Inorganic particles are being studied due to their size and their benefits over chemical agents and medicines currently in the market for chemical imaging, as well as potential tools for disease treatment. Inorganic non-materials are widely used for cellular delivery due to their versatile choices like wide convenience, moneyed usefulness, wise compatibility, and additionally the flexibility of targeted drug delivery and controlled release of drugs (Sharma et al. 2019).

\section{b) Metal-oxide-based nanoparticles:}

A good example would be iron ( $\mathrm{Fe}$ ) nanoparticles that oxidize directly to iron compound $\left(\mathrm{Fe}_{2} \mathrm{O}_{3}\right)$ in the presence of oxygen at room temperature, which will increase their reactivity. Since metal compound nanoparticles have additionally accumulated reactivity and potency, they are synthesized in huge amounts. Aluminum oxide $\left(\mathrm{Al}_{2} \mathrm{O}_{3}\right)$, cerium oxide $\left(\mathrm{CeO}_{2}\right)$, iron oxide $\left(\mathrm{Fe}_{2} \mathrm{O}_{3}\right)$, magnetite $\left(\mathrm{Fe}_{3} \mathrm{O}_{4}\right)$, silicon dioxide $\left(\mathrm{SiO}_{2}\right)$, titanium oxide $\left(\mathrm{TiO}_{2}\right)$, zinc oxide $(\mathrm{ZnO})$ are the foremost unremarkably synthesized materials. As compared to their counter components, these nanoparticles have outstanding characteristics (Sharma et al. 2019).

\section{Carbon-based nanoparticles}

Carbon-based nanoparticles are those created entirely of carbon. Fullerenes, graphene, carbon nanotubes (CNTs), carbon nanofibers, and carbon black and sometimes activated carbon are a number of the categories of carbon nanomaterials that will be classified (Sharma et al. 2019).

\section{Use of AgNPs for pharmaceutical active ingredients}

1. The chemical method of synthesizing rifampicinconjugated silver nanoparticles (Rif-Ag) has been successfully achieved (Farooq et al. 2019).

2. Antifungal drug amphotericin $B$ was used during the AgNP's synthesis as a capping and reducing agent, resulting in AmB-AgNP's antifungal properties (Tutaj et al. 2016).

3. Silver nanoparticles with capped Solanum trilobatum extract have high antimicrobial activity after being synthesized in an eco-friendly way (Ramanathan et al. 2018).

4. Synthesis of silver nanoparticles from Cassia auriculata extracts to prepare bactericidal cold cream against clinical pathogens (Sahana et al. 2014).

5. Discs made from poly(methyl methacrylate) [PMMA] and PMMA nanoparticles were synthesized, while control discs used commercial acrylic resin "Nature-Cryl" (Acosta-Torres et al. 2012).

\section{Disadvantages of silver nanoparticles}

1. Despite the potential of nanodrugs, safety worries are growing. The toxicity of nanodrugs is not completely understood, but researchers are working to improve their understanding. The use of nanoparticles is thought to reduce the toxicity of chemotherapeutic medications and other drugs with narrow therapeutic indexes; however, in vitro and in vivo studies have shown that some nanoparticles are cytotoxic and cause allergies or inflammation in biological systems (Ai et al. 2011).

2. Inhalation of AgNPs has been linked to asthma, bronchitis, emphysema, lung cancer, and neurological illnesses. The gastrointestinal tract is also involved in Crohn's disease and colon cancer (Buzea et al. 2007)

3. Several autoimmune illnesses, including systemic lupus erythematosus, scleroderma, and rheumatoid arthritis, have been linked to AgNPs that reach the circulatory system (Buzea et al. 2007)

4. AgNPs are thought to be particularly hazardous to humans due to their potential toxicity. Skin that has been exposed to AgNP develops a bluish tone. Biological toxicity has been studied in other research as well, such as oxidative damage (McShan et al. 2014) 
5. Nanoparticles produce reactive oxygen species (ROS) and free radicals that cause oxidative stress, inflammation, DNA damage, multiple nucleate development, and fibrosis (Vega-Villa et al. 2008)

6. Argyria, an irreversible discoloration of the skin and eyes caused by excessive silver deposition, has hindered the use of silver as an antibacterial agent due to its potential harmful consequences (Voorde et al. 2005)

7. AgNPs also damage cells, causing DNA damage, activation of antioxidant enzymes, depletion of antioxidant molecules (e.g., glutathione), binding and disabling of proteins, and damage to the cell membrane (McShan et al. 2014)

\section{Various strategies for synthesis of silver nanoparticles}

In addition to physical, chemical, and biological strategies, green synthesis is additionally used to synthesize AgNPs. Figure 2 shows the fundamental realistic diagram of the AgNPs synthesis outline. Top-down" and "bottom-up" AgNP syntheses are the two most frequent techniques. Mechanical grinding of silver bulk may be a top-down methodology. Bottom-up strategies involve chemical reduction, sonodecomposition, and electrochemical methods (Slepička et al. 2020).

Bottom-up and top-down strategies are employed to synthesize nanoparticles. Figure 3 depicts the top-down and bottom-up approaches for the synthesis of nanoparticles. The bottom-up process involves the atom-by-atom construction of nanostructures (like a molecule) and the precipitation or condensation of product material dissolved in solvents, as well as the subsequent separation

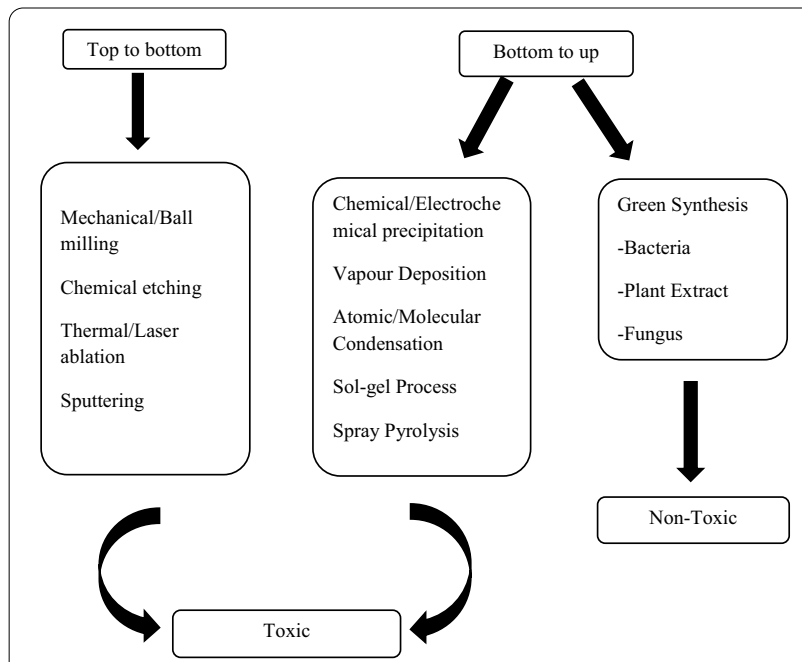

Fig. 2 The fundamental realistic diagram of the AgNPs synthesis outline

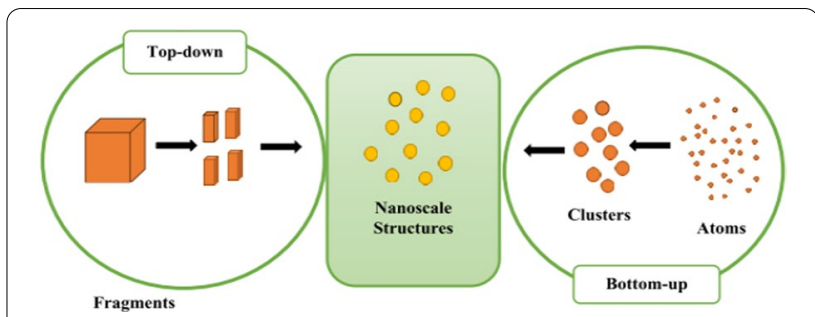

Fig. 3 Top-down and bottom-up approaches for synthesis of nanoparticles

of undesirable solvents. The top-down strategy is based on reducing the size of larger particles using equipment designed to remove material from the "bulk" to build nanoscale structures (Slepička et al. 2020).

\section{Advantages of green synthesis over chemical synthesis of silver nanoparticles (Moosa et al. 2015)}

1. Using this method is environmental friendly

2. Cost-effectiveness is a major advantage.

3. Huge synthesis may be feasible with these emerging technologies.

4. You do not need to use high-pressure, high-energy, high-temperatures, or toxic materials.

\section{Nano-based drug delivery systems}

The inclusion of nanosized particles in cosmetic formulations may increase the stability of active substances including vitamins, unsaturated fatty acids, and antioxidants, hence enhancing their therapeutic potential (Pithawala and Jain 2021). Researchers are particularly interested in metallic nanoparticles because of their potential use in medicine, cosmetics, food, paint, and the textile industry (Legaspi and Fundador 2020). Despite the advantages, pharmaceutical companies are hesitant to invest more in natural product-based drug development and drug delivery systems, preferring instead to search existing chemical compound libraries for new treatments. Natural drugs have specific advantages such as reduced toxicity and adverse effects, low cost, and significant therapeutic potential. In vivo instability, limited bioavailability and solubility, poor absorption in the body, challenges with target-specific delivery and tonic effectiveness, and potential adverse pharmacological effects all complicate the use of large-sized materials in drug administration. By implementing novel drug delivery systems that target treatments to specific body locations, it might be possible to address these critical concerns the act of releasing or delivering drugs (Patra et al. 2018). 
i. Transdermal drug delivery system: The primary purpose of delivery systems is to reach and often pass through the organ of interest. Scientists have recently developed a slew of nanocarriers to aid in medicine delivery into the skin and across biological membranes (Escobar-Chávez et al. 2012). A transdermal medication delivery device permits a controlled release of a confined substance into the systemic circulation by permeation through skin layers. These systems are simple to install and uninstall as needed. In transdermal medication administration, the use of herbal penetration enhancers that penetrate human skin and reduce barrier resistance is generally accepted. Aloe vera (Aloe barbadensis Miller) gel, for example, has been found to improve the penetration of certain medication molecules through epidermal membranes. The physical look of transdermal films is part of their characteristics (color, clarity, completeness, uniformity, surface texture, and flexibility). The film's thickness, folding endurance, flatness studies, weight fluctuation, moisture content, percentage moisture uptake hardness, tensile strength, and percent elongation of the substance of the drug, stability research, in vitro drug release studies, in vitro drug release research, and in vitro skin permeation studies are all examples of in vitro drug release studies (Sharma 2016).

ii. Cream: To address diverse skin diseases and infections, novel dermatological and cosmetic formulations with antibacterial and antifungal properties are used (Sonia et al. 2017). Topical medications that can be applied to the skin are known as creams. Creams are "viscous liquid or semi-solid emulsions of either the oil-in-water or water-in-oil type," according to the definition. The consistency of the dose forms differs depending on whether they are made of oil or water. These topical formulations are used to transfer drugs into the underlying layer of the skin or the mucous membrane for a localized effect. For skin disorders, a specialized drug administration into the skin is required (Chauhan and Gupta 2020). Creams can be ayurvedic, herbal, or allopathic which are used by people according to their needs for their skin conditions. They contain one or more drugs substances dissolved or dispersed in a suitable base. Creams may be classified as $\mathrm{o} / \mathrm{w}$ or w/o type of emulsion based on phases (Sahu et al. 2016). The assessment of silver nanoparticle-incorporated cream involves physicochemical attributes that include grittiness, $\mathrm{pH}$, and rheological property, emulsion, and stability tests (Pithawala and Jain 2021). iii. Gel: According to the USP, gels are semisolids made up of minute inorganic particles floating in a liquid or large organic molecule interpenetrated by liquid. Gels are clear or translucent semisolid compositions with a high solvent/gelling agent ratio. In comparison with creams and ointments, gels frequently give a faster release of pharmacological material, regardless of the medication's water solubility. They are highly biocompatible, with a lesser chance of irritation or bad reactions, and they are simple to apply and remove. Thixotropic, greaseless, readily spreadable, quickly removed, emollient, non-staining, compatible with a variety of excipients, and water-soluble or miscible are only a few of the benefits of dermatological gels. Different features of the gel can be tested, including $\mathrm{pH}$, spreadability, extrudability, viscosity, swelling characteristics, in vitro drug diffusion, and drug release kinetic investigations (Prusty and Parida 2014).

iv. Nanoemulsions: Nanoemulsions, also known as submicron emulsions, ultrafine emulsions, and mini-emulsions, are isotropic dispersions made up of two immiscible liquids, such as water and oil, stabilized by an interfacial film made up of an appropriate surfactant and co-surfactant to create a single phase (Gurpreet and Singh 2018). It does not form on its own; it requires external shear to break up bigger droplets into smaller ones (Panda et al. 2017). Two approaches for making nanoemulsions are persuasion and brute force. Various characterization approaches for nanoemulsions include entrapment efficiency, particle size, polydispersity index, zeta potential, and characterization using differential scanning calorimetry, as well as Fourier transform infrared spectroscopy and transmission electron microscopy. Furthermore, drug release, in vitro permeability, stability and thermodynamic stability, shelf life, dispersibility, and surface tension, as well as $\mathrm{pH}$ and osmolarity are all examined in vitro (Gurpreet and Singh 2018).

v. Nanosuspension: A biphasic system consisting of pure drug particles dispersed in an aqueous vehicle with a diameter of fewer than $1 \mathrm{~m}$ and stabilized by surfactants, nanosuspension is a biphasic system consisting of pure drug particles dispersed in an aqueous vehicle with a diameter of fewer than $1 \mathrm{~m}$ and stabilized by surfactants. They can be used to increase the solubility of medications that are not well soluble either in water or fat. As a result, the rate of flooding of the active component rises, resulting in a faster reach of the maximum plasma level. Nanosuspension has several advantages over traditional suspensions, including increased disso- 
lution velocity, drug saturation solubility, enhanced biological performance, long-term physical stability, and ease of fabrication (Panda et al. 2017).

\section{Taxonomy and chemical constituents of Aloe vera and Thuja orientalis plant \\ Aloe vera:}

Scientific name: Aloe barbadensis Miller.

Synonyms: Aloe perryi Baker, Aloe vera Linn or Aloe barbadensis Mil and Aloe ferox Miller.,

Biological source: Aloe is the dried juice collected by incision, from the bases of the leaves of various species of Aloe.

Family: Liliaceae.

Chemical constituents: Aloe vera contains more than 75 different compounds, including vitamins (vitamin A, C, E, and B12), enzymes (i.e., amylase, catalase, and peroxidase), minerals (i.e., zinc, copper, selenium, and calcium), sugars (monosaccharides such as mannose6-phosphate and polysaccharides such as glucomannans), anthraquinones (aloin and emodin), fatty acids (i.e., lupeol and campesterol), hormones (auxins and gibberellins), and others (i.e., salicylic acid, lignin, and saponins) (Sánchez et al. 2020).

Aloe vera as shown in Fig. 4 is a semi-tropical plant. Throughout history, it has been described as an all-purpose herb with notable accomplishments. The plant's thorny, tapered leaves are held up by short shoots near the surface of the soil or soil surface. Fresh gel is usually recommended because some of the active ingredients in the gel seem to diminish over time. An adhesive-like substance forms on the skin when Aloe vera gel is applied. This acts as a natural "band-aid," keeping nutrients in and preventing bacteria or agents from prohibiting healing. Aloe vera gel also contains a significant amount of water, which is essential for the body's recovery process.

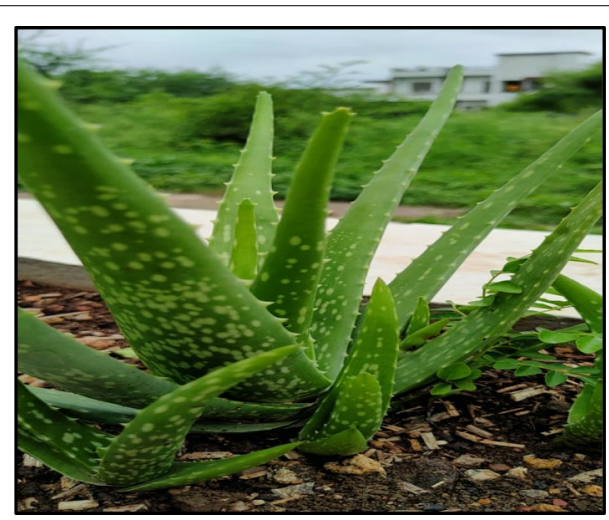

Fig. 4 Aloevera plant
Traditional healers' uses that are not supported by experimental or clinical evidence include the treatment of skin disorders, hemorrhoids, psoriasis, anemia, glaucoma, petit lesion, tuberculosis, blindness, seborrheic eczema, and fungus infections. As well as being effective when taken orally, Aloe vera can be used topically as an ointment, cream, or lotion. Dermo therapeutic use of Aloe vera is widespread because it is an astringent, body lotion, humidifier, and cleanser. It softens the skin, lowers wrinkles, and cures acne, herpes, red spots, psoriasis, eczema, mycosis, fever blisters, skin irritation, and protects the skin from pollution. Aside from that, it is ideal for curing sunburns, fragile skin, and removing dead skin cells and skin. Most of the amino acids and vitamins that our skin needs to heal are found in Aloe vera, making it an excellent healing agent.

Herbal penetration enhancers, in particular, have received a lot of attention, and Aloe vera, an ostensibly skin-friendly and humectant product, is one such enhancement system. While there is some evidence suggesting that Aloe vera enhances skin penetration, only one paper mentions its use as a vehicle for various substances. As a result of two recent US patent filings, Aloe vera is credited with increasing the skin penetration of co-formulated treatments.

Aloe seems to increase penetration as:

- It penetrates deep into the epidermis.

- It maintains the skin's acid-alkaline pH balance.

- Bacteria, viruses, and fungi are prevented from multiplying.

- It is an anti-inflammatory and astringent, and it is also a natural preservative.

- In addition, it provides pain relief and itching relief.

- It helps to stay the skin hydrated and healthy.

- It dilates the blood vessels under the skin and speeds up the blood flow through the system.

- In addition, it promotes cell division and accelerates tissue healing.

Aloe vera contains lignin, which allows it to penetrate the cellular level and have positive benefits. This plant also contains saponin, a natural cleaner. Both of these components must work together to accomplish cellular penetration (Sharma et al. 2015).

Aloe vera is recognized to possess several medicinal properties since thousands of years ago. It contains treasures of nutritional and antipathogenic compounds. Aloe vera is opted for the synthesis of silver nanoparticles due to the presence of natural phytochemicals which offer natural capping and reducing agents. Aloe vera extracts are used for the synthesis of stable many studies that have looked at the bactericide, antifungal, and mosquitocidal 
properties of AgNPs. Aloe extracts contain compounds that cause steric repulsion between individuals, which prevents nanoparticles from aggregating. Using Aloe vera as a surface-active agent prevents nuclei aggregation by decreasing the overall surface energy result of it contains a large number of chemical constituents (Vélez et al. 2018).

Thuja orientalis: Thuja plant is shown in Fig. 5.

Scientific name: Thuja orientalis.

Synonyms: Morpankhi, Oriental thuja, Oriental arborvitae, Vidya plant.

Family: Cupressaceae.

Chemical constituents: Rhodoxanthin, amentoflavone, hinokiflavone, quercetin, myricetin, carotene, xanthophylls, and ascorbic acid are all found in conifer leaves. per Nickavar et al., nineteen and twenty-eight compounds were found among the volatile oils of the fruit and leaf, severally, with the fruit oil containingpinene (52.4\%), 3-carene (14.2\%), -cedrol (6.5\%), and phellandrene $(5.1 \%)$, and thus the leaf oil containing -pinene (21.9\%), -cedrol (20.3\%), 3-carene (10.5\%), and hydrocarbon (7.2 Thujone may well be associated organic compound and monoterpene that comes in a pair of diastereomeric forms in nature: -thujone and -thujone (Srivastava et al. 2012).

Uses: It is used to treat bronchial inflammation, enuresis, cystitis, psoriasis, female internal reproductive organ carcinomas, amenorrhea, and rheumatism in several types of ancient medication like folk medication and homeopathy. The herb Thuja is also used to treat skin, blood, digestive system, renal, and brain diseases, as well as wart-like excrescences and spongy tumors. Various ailments such as coughs, hemorrhages, excessive menstrual flow, bronchitis, asthma, skin infections, anemia, rheumatic aches, and premature blandness are all treated with Oriental arborvitae. Hair development is assumed to be assisted by their use. Antipyretic, astringent, diuretic, emmenagogue, emollient, expectorant, refrigerant, and

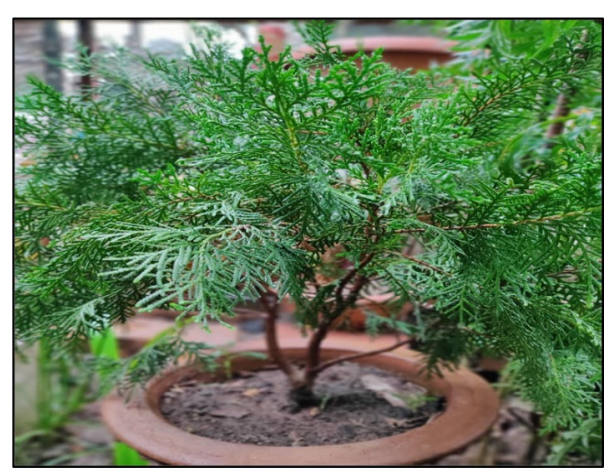

Fig. 5 Thuja plant stomachic are all properties of the leaves (Srivastava et al. 2012).

Thuja orientalis (also known as Morpankhi) is a coniferous tree genus that belongs to the Cupressaceae family. $T$. Orientalis is a monoecious, evergreen tree, or shrub that reaches a height of 10-60 feet. There are five species in the genus, two of which are endemic to North America and three to eastern Asia. Vidya plant, scientific name Thuja, is a non-flowering, seed-bearing evergreen garden shrub native to India (Srivastava et al. 2012).

It is an evergreen coniferous tree used in landscaping, with a shallow cup in its early years that widens as it becomes older, a conic overall shape, and laminar branching. The branches are fan-like in appearance and are disposed of upwards. Branches and leaves are flat in shape. Every branch changes color from deep green to green yellowish color as it gets closer to the tip. It is a dioic tree, with female cones that are whitish or rose pale and later bluish-green, with well-marked tips that open like spikes, each of which is a squama and has 6-8 flattened oval form, are thick, and have an apical hook, the cone is dehiscent when mature and turns brownish-tan. Every cone contains about 6 ovoid-trigonoidas seeds and is expelled wingless. The leaves are flattened fan-shaped and have resin glands on them. Essential oils found in the leaves of these plants are used to cure fungal infections, cancer, moles, and parasitic worms (Hernández and Arenas 2016). The essential oil that is extracted from the leaves is poisonous. $\alpha$-Thujone is an insecticide and antihelminthic substance that can be used to treat parasitic worms. $\alpha$-Thujone, on the other hand, is a poisonous chemical that interferes with neurological signals in the brain (Sharma and Sharma 2016).

\section{Methodology}

\section{Aloe vera extract preparation}

Fresh Aloe vera leaves were collected and rinsed many times with distilled water to remove dirt particles, before being sun-dried to remove any remaining moisture. In a 500-ml glass beaker, weigh 10-g dried aloe leaves crumpled and shredded with a glass rod, add $200 \mathrm{ml}$ sterilized distilled water to a $250 \mathrm{ml}$ beaker, and boil for $20 \mathrm{~min}$, or until the color of the aqueous solution changes to yellow. After cooling the extract, it was filtered through Whatman filter paper, centrifuged for half an hour at 3000 RPM, and the supernatant layer was pipette separated. The aloe extract should be kept at a temperature of $-15{ }^{\circ} \mathrm{C}$ (Khan et al. 2017).

\section{Thuja leaves extract preparation}

Five gram finely shredded leaves were weighed into a flask with $100 \mathrm{ml}$ of deionized water and dropped at a temperature of $80-85{ }^{\circ} \mathrm{C}$. The mouth of the flask was 
capped to prevent evaporation losses for $15 \mathrm{~min}$. The mixture was cooled for $5 \mathrm{~min}$, then filtered with Whatman 40 filter paper, and stored in amber bottles at a low temperature (Hernández and Arenas 2016).

\section{Preparation of silver nanoparticles (AgNPs) by using Aloe vera leaves extract}

Prepare a $0.001 \mathrm{M} / 1 \mathrm{mM} \mathrm{AgNO}_{3}$ solution by dissolving $0.17 \mathrm{~g}$ silver nitrate in a $1000-\mathrm{ml}$ volumetric flask with distilled water and increasing the volume to $1000 \mathrm{ml}$. Twenty milliliters of aloe leaf extract was added dropwise to a $50 \mathrm{ml} 0.001 \mathrm{M} \mathrm{AgNO}_{3}$ solution with continuous shaking at room temperature until the solution becomes gray-black. The reduction of silver ions by the liquid plant extract, as well as the production of stable silver nanoparticles, resulted in a gray-black color (Khan et al. 2017).

\section{Synthesis of AgNPs by using Thuja leaves extract}

At varied time intervals, $50 \mathrm{ml}$ of Thuja orientalis was added to $10 \mathrm{ml}$ of $\mathrm{AgNO}_{3}$ with steady stirring. The production of silver nanoparticles was shown by the color change from yellow to amber. In a $100 \mathrm{~mL} \mathrm{AgNO}_{3}$ solution, $10 \mathrm{~mL}$ of Thuja orientalis leaf extract was added. Due to the surface, the color changed from pale yellow to brown. After $24 \mathrm{~h}$ of incubation, the color of the experimental sample (a mixture of silver nitrate and leaf extract) turned dark brown and then darker. The material was centrifuged at $9000 \mathrm{rpm}$ for $20 \mathrm{~min}$ at $0-4{ }^{\circ} \mathrm{C}$ after the color change indicated the creation of silver nanoparticles. The supernatant was then separated, and the precipitate was generated and water was used to wash away the precipitate that had collected at the bottom. The precipitate was filtered and air-dried, yielding a brown color powder. It was utilized in later research to determine characteristics such as the size and form of silver nanoparticles (Hernández and Arenas 2016).

\section{Mechanism of action}

Silver has become one of the most studied oligodynamic materials due to its wide variety of bactericidal effects, efficacy, low toxicity, and multiple applications as a disinfectant. The oligodynamic effect is a term used to describe the biocidal effects of metals, particularly heavy metals, at low concentrations. Silver nanoparticles (AgNPs) have been shown to have an oligodynamic effect due to their large surface areas and ability to bind with microorganism biomolecules, penetrate cells, generate reactive oxygen species (ROS) and free radicals, and act as modulators in microorganism signal transduction pathways (Prasher et al. 2018). The wide range of oligodynamic materials, particularly AgNPs, has sparked interest as a technique to accelerate the development of nanotherapeutics such as antibiotics. Surface alteration of AgNPs may affect the oligodynamic effect of nanoparticles, as surface modification can stabilize these nanoparticles (e.g., AgNPs modified with polyimide). The half-encapsulation of AgNPs with amorphous polyimide has been found to improve silver stability while simultaneously increasing bactericide action.

Strong adhesion to E. coli and Bacilli has been seen using polydopamine-coated glass spheres with robust incised glass spheres (PDA-HF/GSs). As a result, the production of biofilms is inhibited. Using a solution casting method, the metal oxide was added to AgNPs, resulting in a homogeneous dispersion of nanoparticles. These modified nanoparticles inhibit the growth of E. coli and Staphylococcus aureus as well as the formation of the film. AgNPs have significantly higher antibacterial effects than other metallic nanoparticles and are less harmful to cells. Although some metallic nanoparticles, such as copper nanoparticles, exhibit more bactericidal activity than AgNPs, they are toxic to cells (CuNPs). Because their mode of action requires bacterial membrane rupture and photocatalytic electron transfer, CuNPs have a higher ability to limit microorganism development than AgNPs. Despite their superior antibacterial action, CuNPs are less stable than CuONPs and have a higher chance of converting to $\mathrm{CuONPs}$ by oxidation. Because gold nanoparticles (AuNPs) lack an apparent intrinsic bactericide effect, they have a lower ability to suppress microbe formation than AgNPs. To cling to the bacterial surface, they rely solely on electrostatic forces. The mechanism of action is determined by nanoparticle size; the smaller the nanoparticles, the lower the minimum inhibitor concentration (MIC). AgNPs have four antibacterial properties: (a) adhesion to microorganisms' surface membranes. (b) AgNPs penetrating cells, disrupting biomolecules, and causing intracellular damage, (c) inducing cellular toxicity by producing ROS, causing oxidative stress in cells, and (d) disrupting cell signal transduction pathways

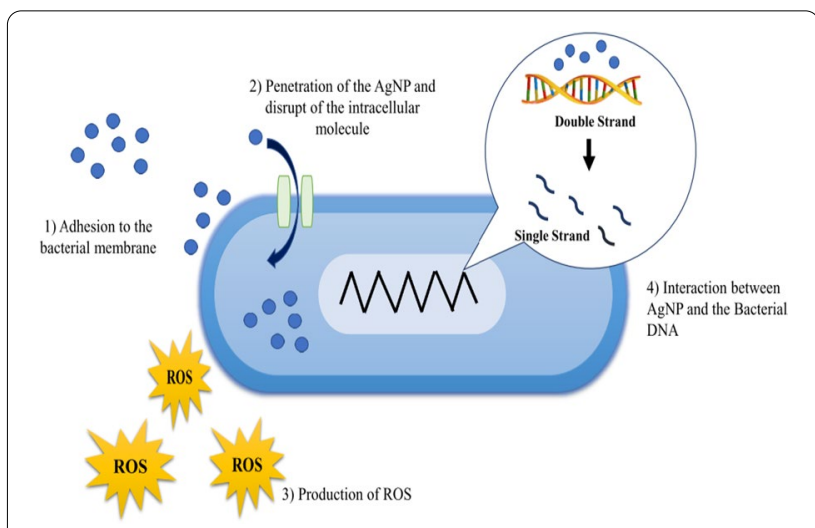

Fig. 6 The general mechanism of action in bacteria 
(Salleh et al. 2020). The common mechanism of action in bacteria is depicted in Fig. 6

\section{Characterization techniques}

Evaluation of silver nanoparticles (AgNPs) involves varied sorts of analytical techniques which includes $\mathrm{X}$-ray diffractometry (XRD) at the wavelength $(\lambda)=1.54056 \AA$, $\mathrm{X}$-ray photoelectron spectroscopic analysis (XPS) typically between 300 and $400 \mathrm{eV}$, Fourier transform infrared spectroscopy (FTIR) in the wavelength range of $400-4000 \mathrm{~cm}^{-1}$, ultraviolet-visible spectroscopic analysis (UV-Vis spectroscopy) at the range of $400-500 \mathrm{~nm}$, transmission electron microscopy (TEM), scanning electron microscopy (SEM), dynamic light scattering (DLS) at a scattering angle of $173^{\circ}$, and localized surface plasma resonance (LSPR) at the wavelength of $300-1100 \mathrm{~nm}$ (Alheety et al. 2019). These analyses are necessary to assess the behavior, biodistribution, and reactivity of these fabricated nanoparticles. Table 1 shows the fundamental functions of the analytical techniques used for the characterization of AgNPs. However, the results of these analytical techniques depend on the synthesis of AgNPs that conjointly alter the physicochemical properties of the nanoparticles (Pourzahedi and Eckelman 2015).

\section{Antifungal activity of synthesized AgNPs by disc diffusion method \\ Source of organism and growth media composition}

Broth preparation Two drops of antibiotics were added to $1.3 \mathrm{~g}$ of nutritional broth in a hundred milliliters of distilled water. The conical flask was cotton-wrapped and autoclaved for $15 \mathrm{~min}$ at $15 \mathrm{~b} / \mathrm{b}$ inch 2 pressure and $121{ }^{\circ} \mathrm{C}$ (Medda, et al. 2015).

Inoculation Fungi were inoculated with a needle from a pure culture medium into the broth medium and incubated at $30^{\circ} \mathrm{C}$ in an incubator for $72 \mathrm{~h}$ after cooling the broth medium (Medda, et al. 2015).

\section{Medium preparation and antifungal activity test}

Ten gram dextrose monohydrate and $14 \mathrm{~g}$ nutritional agar were cooked with $500 \mathrm{ml}$ of potato extract (10$12 \%)$; 3-4 drops of antibiotic were added to prevent bacterial development, and the $\mathrm{pH}$ of the solution was kept between 5 and 5.6. The agar media were then placed into sterile Petri plates, and $50 \mathrm{l}$ of fungal broth culture was spread on each plate with the use of a spreader once it had solidified. Each plate was then drilled with a hole borer. In each hole of the plate, $100 \mathrm{l}$ AgNPs solution, leaf extract only, and leaf extract and salt solution were poured and stored for $48 \mathrm{~h}$ at a $30^{\circ} \mathrm{C}$ temperature for further observation (Medda et al. 2015).

\section{Microscopic observation}

The silver nanoparticle's antifungal activity was evaluated using a light microscope. The inhibition zone is to determine antifungal activity. AgNPs' antifungal activity, on the other hand, is influenced by the type of fungus as well as the size of AgNPs and is associated with the formation of pits in microorganism cell walls. AgNPs bind to the membranes of fungal cells, causing the membrane potential to be disturbed (Medda et al. 2015).

Table 1 Fundamental functions of the analytical techniques used for the characterization of AgNPs

\begin{tabular}{|c|c|}
\hline Analytical techniques & Functions \\
\hline X-ray diffraction & $\begin{array}{l}\text { Measure the degree of crystallinity at the atomic scale. Used to analyze the structure of nanoparticles, particle } \\
\text { sizes, for compounds identification, and to determine structure imperfections in the structures. The analysis } \\
\text { depends on the formation of diffraction patterns (Alheety et al. 2019) }\end{array}$ \\
\hline X-ray photoelectron spectroscopy & $\begin{array}{l}\text { Determine the electronic states by atoms which include the oxidation state, and electron transfer in the nano- } \\
\text { particles. Estimate the empirical formulae by surface chemical analysis. Characterize the nanoparticles' surface } \\
\text { in the liquid forms (Pourzahedi and Eckelman 2015) }\end{array}$ \\
\hline Fourier transform infrared spectroscopy & Characterize various chemical bonding in nanomaterials (Pal et al. 2017) \\
\hline UV-Vis spectroscopy & $\begin{array}{l}\text { Evaluate the stability and characteristics of AgNPs. Absorption of AgNPs depends on the dielectric medium, } \\
\text { particle size, and the chemical environment. Size depends on surface plasmon for metal nanoparticles rang- } \\
\text { ing from } 2 \text { to } 100 \mathrm{~nm} \text { (Nguyen et al. 2018) }\end{array}$ \\
\hline Transmission electron microscopy & $\begin{array}{l}\text { Measure of particle size, morphology, and size distribution. Provide better spatial resolution compared to SEM } \\
\text { (Baudot et al. 2010) }\end{array}$ \\
\hline Scanning electron microscopy & $\begin{array}{l}\text { Evaluate the morphology of AgNPs. Histogram obtains from images. Manually measure and count the parti- } \\
\text { cles or using specific software (Gorham et al. 2012) }\end{array}$ \\
\hline Dynamic light scattering & $\begin{array}{l}\text { Measure nanoparticles size. Evaluate their stability over time in suspension at different pH and temperature } \\
\text { conditions (Balaji et al. 2009) }\end{array}$ \\
\hline Localized surface plasmon resonance & $\begin{array}{l}\text { Determine spatial oscillation of non-excited or excited (near-visible light) electron. Evaluate the molecular } \\
\text { interaction on the surface of a nanoparticle. Depends on several factors: particle's size and shape electronic } \\
\text { properties, dielectric media, and temperature (Chen et al. 2017) }\end{array}$ \\
\hline
\end{tabular}




\section{Antimicrobial activity}

The antimicrobial activity of produced silver nanoparticles of Aloe vera and Thuja orientalis leaf extract was investigated using the disc diffusion method against a variety of microorganisms. The highest zones of inhibition against Bacillus subtilis, Staphylococcus aureus, Salmonella typhi, and Escherichia coli were identified in both Aloe vera and Thuja orientalis silver nanoparticles.

Bacillus subtilis, Staphylococcus aureus, Salmonella typhi, and Escherichia coli were injected with a needle from a pure culture medium into the broth medium and stored in an incubator at $30{ }^{\circ} \mathrm{C}$ for $72 \mathrm{~h}$ after cooling the broth medium (Balouiri and Sadiki 2016).

\section{Disc diffusion method}

The antifungal activity of the samples was tested using nutrient agar media. In distilled water, prepare a medium with a particular concentration $(13 \mathrm{~g} / \mathrm{L})$. Sterilize the media and Petri plates, then pour the media into the Petri plates and let it set. After the media have solidified, wipe the bacterial culture on solid plates with sterilized cotton, and sterilized forceps were used to place discs with various concentrations $(15,20,25$, and $30 \mathrm{l})$ on the medium. Incubate the plates in an incubator at $37^{\circ} \mathrm{C}$ for $24 \mathrm{~h}$, then measure the diameter of the zone of inhibition, which defines the growth of bacteria and the activity of that precise concentration of synthesized silver nanoparticles.

Because it is impossible to quantify the amount of antimicrobial agent diffused into the agar medium, the agar disc diffusion method is not suited for determining the minimum inhibitory concentration (MIC). Nonetheless, the disk diffusion method has a number of advantages over other approaches, including the convenience of use, low cost, the capacity to test a large variety of bacteria and antimicrobial drugs, and the ease with which the data can be interpreted.

The disc diffusion and broth or agar dilution procedures are the most well-known and basic approaches. In vitro antimicrobial activity can be assessed using a variety of laboratory approaches. Time-kill tests and flow cytofluorometric methods are used to further investigate an agent's antimicrobial effect. Time-dependent or concentration-dependent cellular damage induced by the test microorganism is also determined by these methods (Balouiri and Sadiki 2016).

\section{Time-kill test (time-kill curve)}

Testing for bactericidal or fungicidal effects using the time-kill approach is the most acceptable way to do so. Antimicrobial susceptibility testing is an important approach for getting information on the dynamic interaction between an antibacterial agent and its target microbe. In the time-kill test, antimicrobial activity is determined by either time or concentration.

A final concentration of $0.25 \mathrm{MIC}$ and $1 \mathrm{MIC}$ is used in the first and second tubes, respectively. The third tube is used as growth control. The incubation period varies $(0$, $4,6,8,10,12$, and $24 \mathrm{~h}$ ). When the number of living cells $(\mathrm{CFU} / \mathrm{mL})$ in each tube is determined using an agar plate count method, the proportion of dead cells compared to the growth control is calculated. $90 \%$ lethality for six hours is similar to $99.9 \%$ lethality for $24 \mathrm{~h}$. Synergism or antagonism between drugs can also be determined using this method (Balouiri and Sadiki 2016).

\section{Applications of silver nanoparticles}

AgNPs have been widely used in household tools, the healthcare industry, food storage, environmental, and biomedical applications due to their outstanding features. AgNPs have also been widely used in medical applications such as cardiovascular implants, catheters, orthopedic implants, and dental composites as an antibacterial layer.

1. The importance of AgNPs in the food industry: Because of their antibacterial activity, AgNPs are frequently employed in the food business as preservatives (Cushen et al. 2012). The smallest amount of AgNPs is safe for humans but fatal to the majority of viruses and bacteria, making them helpful for food and water sanitization in everyday life and as an infection resistor in medicine. Sunriver industrial nanosilver fresh food bag is one of the commercially available bags with silver nanoparticles is the nanosilver fresh food bag (Huang et al. 2011). Because of their anti-fungicidal and anti-bacterial properties, AgNPs are widely utilized in everyday products such as soaps, food, plastics, pastes, and textiles.

2. Food packaging and nanotechnology: A good packaging material should have gas and moisture permeability, as well as strength and biodegradability (Couch et al. 2016). From offering better packaging material with higher mechanical strength, barrier qualities, and antimicrobial coatings to nanosensing pathogen detection and detection, nano-based "smart" and "active" food packaging provides various advantages over conventional packaging methods. The antimicrobial coatings for nanosensing pathogen detection and warning consumers about food safety (Mihindukulasuriya and Lim 2014). Food packaging can benefit from nanocomposites as an "active material" for packaging and material coating (Pinto et al. 2013). 
3. Disinfection of groundwater and biological wastes: Researchers looked at the effect of Ag-NPs on microbial communities in wastewater treatment plants and discovered that original wastewater biofilms are very resistant to Ag-NP treatment. After 24 h, the reduction in biofilm microorganisms evaluated by heterotrophic plate counts was negligible when $200 \mathrm{mg}-1$ Ag-NPs were applied. When bacteria are exposed to Ag-NP, biofilms can protect them physically and extracellular polymeric substances (EPS) may play an important role in this protection (Verma and Maheshwari 2019).

4. Implants for the cardiovascular system: A prosthetic silicone heart valve was the first cardiovascular device to be coated with the silver element to lessen the risk of endocarditis. The use of silver was supposed to reduce the heart's inflammatory response by preventing bacterial infection on the silicone valve. Clinical experiments of the silver heart valve, on the other hand, discovered that it produces hypersensitivity, inhibits normal fibroblast function, and causes paravalvular leakage in patients (Murphy et al. 2015). As a result, efforts have focused on introducing AgNPs into medical devices as a viable antibacterial coating that is both safe and non-toxic.

5. Catheters: Catheters used in hospitals have a significant risk of infection, which can result in unwelcome problems. As a result, AgNPs have been studied as a way to reduce biofilm formation on catheters. Polyurethane catheters with a coat of AgNPs have recently been modified to make efficient antibacterial catheters. Several studies have found that AgNP-coated catheters can effectively suppress germs in animal models for up to $72 \mathrm{~h}$ (Paladini et al. 2013). Furthermore, the AgNP-coated catheter was found to be harmless in a 10-day in vivo trial in mice.

6. Dentistry: Dental instruments and bandages have also been treated with AgNPs. Incorporating AgNPs into orthodontic adhesives could improve or sustain an orthodontic adhesive's shear bond strength while also boosting its bacterial resistance (Akhavan et al. 2013). Incorporating AgNPs into dental composites was found to minimize microbial colonization of lining materials while also improving antifungal efficiency (Chladek et al. 2013). AgNPs added into endodontic fillings had a similar antibacterial activity on Streptococcus miller, S. aureus, and E. faecalis (Magalhães et al. 2012).

7. Implants and fixtures for orthopedic and orthodontic surgery: Bacterial infections associated with the implant and joint replacements are common (1.0-4.0\%) and are one of the most dangerous consequences in orthopedic surgery because they are dif- ficult to treat and result in increased morbidity and significantly worse results. As a result, AgNPs have been added to plain poly(methyl methacrylate) bone cement, which is used to hold joint prostheses during hip and knee replacement surgery to diminish bacterial resistance. In orthopedic and orthodontic surgery, our team looked explored adding AgNPs into PLGA grafts as a viable implant and fixation material. All of the investigations found that there was a considerable increase in antibacterial activity without any notable harm (Liu et al. 2012).

\section{Conclusions}

The presence of nanoparticles, particularly metallic nanoparticles, has been recognized worldwide and is becoming extremely valuable in a variety of sectors. This review covers a wide range of research on silver nanoparticles to gain a better understanding of their physicochemical feature characterization, production, mechanisms of action, and applications. Various AgNP factors, such as size, surfactant, and structural shape, influence the unique physicochemical properties of these nanoparticles. Even though there are a variety of ways to make AgNPs, green synthesis has a high yield and biocompatibility because it uses natural agents and harmless chemicals. In this paper, we describe the green manufacture of silver nanoparticles utilizing Aloe vera and Thuja orientalis leaf extracts, as well as the method to test their antifungal activity.

\section{Abbreviations}

AgNPs: Silver nanoparticle; LSPR: Localized surface plasma resonance; SEM: Scanning electron microscopy (SEM); DLS: Dynamic light scattering; XRD: X-ray diffractometry; CuNPs: Copper nanoparticles; ROS: Reactive oxygen species.

\section{Acknowledgements}

Authors are really thankful to the P. R. Pote Patil College of Pharmacy, Amravati, for helping us to build.

\section{Authors' contributions}

Format required as per the Journal is framed by PJB. He was a major contributor in compiling and writing the manuscript. Manuscript review and content specification is guided by MGT, RAB complies the data as per the format set by the journal. Language editing is done by VRM. Citation in the article is done by VKS. The articles required to collect the information and framing the raw data required for the manuscript preparation are done by SNS, RAS, and RRM. All authors read and approved the final manuscript.

\section{Funding}

Not applicable.

Availability of data and materials Not applicable.

\section{Declarations}

Ethics approval and consent to participate

Not applicable. 


\section{Consent for publication \\ Not applicable.}

\section{Competing interests}

The authors declare that they have no competing interests.

\section{Author details}

'Department of Pharmaceutical Chemistry, P. R. Pote Patil College of Pharmacy, Amravati 444602, India. ${ }^{2}$ Department of Pharmaceutics, P. R. Pote Patil College of Pharmacy, Amravati 444602, India.

Received: 13 August 2021 Accepted: 16 October 2021

Published online: 30 October 2021

\section{References}

Acosta-Torres LS et al (2012) Cytocompatible antifungal acrylic resin containing silver nanoparticles for dentures. Int J Nanomed 7:4777

Ai J et al (2011) Nanotoxicology and nanoparticle safety in biomedical designs. Int J Nanomed 6:1117

Akhavan A et al (2013) Investigating the effect of incorporating nanosilver/ nanohydroxyapatite particles on the shear bond strength of orthodontic adhesives. Acta Odontol Scand 71(5):1038-1042

Alheety NF, Majeed AH, Alheety MA (2019) Silver nanoparticles anchored 5-methoxy benzimidazolthiomethanol (MBITM): modulate, characterization and comparative studies on MBITM and Ag-MBITM antibacterial activities. J Phys Conf Ser 1294(5):052026

Balaji DS et al (2009) Extracellular biosynthesis of functionalized silver nanoparticles by strains of Cladosporium cladosporioides fungus. Colloids Surf B Biointerfaces 68(1):88-92

Balouiri M, Sadiki M et al (2016) Methods for in vitro evaluating antimicrobial activity: a review. J Pharmaceut Anal 6:71-79

Baudot C, Tan CM, Kong JC (2010) FTIR spectroscopy as a tool for nano-material characterization. Infrared Phys Technol 53(6):434-438

Buzea C, Pacheco Il et al (2007) Nanomaterials and nanoparticles: sources and toxicity. Biointerphases 2(4):MR17-MR71

Chauhan L, Gupta S (2020) Creams: a review on classification, preparation methods, evaluation and its applications. J Drug Deliv Therap 10(5-s):281-289

Chen S et al (2017) Graphene quantum dot/silver nanoparticle hybrids with oxidase activities for antibacterial application. ACS Biomater Sci Eng 3(3):313-321

Chladek G et al (2013) Sorption, solubility, bond strength and hardness of denture soft lining incorporated with silver nanoparticles. Int J Mol Sci 14(1):563-574

Couch LM et al (2016) Food nanotechnology: proposed uses, safety concerns and regulations. Agro Food Ind Hi-Tech 27(1):36-39

Cushen M et al (2012) Nanotechnologies in the food industry —recent developments, risks and regulation. Trends Food Sci Technol 24(1):30-46

Escobar-Chávez JJ et al (2012) Nanocarriers for transdermal drug delivery. Res Rep Transderm Drug Deliv 1:3

Farooq U et al (2019) Rifampicin conjugated silver nanoparticles: a new arena for development of antibiofilm potential against methicillin resistant Staphylococcus aureus and Klebsiella pneumoniae. Int J Nanomed 14:3983

Gajbhiye S, Sakharwade S (2016) Silver nanoparticles in cosmetics. J Cosmet Dermatol Sci Appl 6(1):48-53

Galatage ST et al (2020) Silver nano particles by green synthesis: an overview. Res J Pharm Technol 13(3):1503-1510

Gorham JM et al (2012) UV-induced photochemical transformations of citratecapped silver nanoparticle suspensions. J Nanopart Res 14(10):1-16

Gurpreet K, Singh SK (2018) Review of nanoemulsion formulation and characterization techniques. Indian J Pharmaceut Sci 80(5):781-789

Hernández L, Arenas D (2016) Green synthesis, characterization and stabilization of nanoparticles silver with Thuja orientalis extract. J Nanomater Mol Nanotechnol. https://doi.org/10.4172/2324-8777.1000198

Huang Y et al (2011) Nanosilver migrated into food-simulating solutions from commercially available food fresh containers. Packag Technol Sci 24(5):291-297
Khan A et al (2017) Yaqoob (2017) Novel synthesis and characterization of silver nanoparticles from leaf aqueous extract of Aloe vera and their antimicrobial activity. J Nanosci Nano-Technol 1:103

Legaspi DS, Fundador NG (2020) Green synthesis of silver nanoparticles using calabash (Crescentia cujete) fruit extract and their antimicrobial properties. Philippine J Sci 149(1):239-246

Liu Y et al (2012) The antimicrobial and osteoinductive properties of silver nanoparticle/poly (DL-lactic-co-glycolic acid)-coated stainless steel. Biomaterials 33(34):8745-8756

Magalhães APR et al (2012) Nanosilver application in dental cements. Int Sch Res Not 2012:1-6

McShan D, Ray PC, Yu H (2014) Molecular toxicity mechanism of nanosilver. J Food Drug Anal 22(1):116-127

Medda S et al (2015) Biosynthesis of silver nanoparticles from Aloe vera leaf extract and antifungal activity against Rhizopus sp. and Aspergillus sp. Appl Nanosci 5(7):875-880

Mihindukulasuriya SD, Lim LT (2014) Nanotechnology development in food packaging: a review. Trends Food Sci Technol 40(2):149-167

Moosa AA, Ridha AM, Al-Kaser M (2015) Process parameters for green synthesis of silver nanoparticles using leaves extract of Aloe vera plant. Int J Multi Curr Res 3:966-975

Murphy M et al (2015) Current development of silver nanoparticle preparation, investigation, and application in the field of medicine. J Nanomater. https://doi.org/10.1155/2015/696918

Nguyen L et al (2018) X-ray photoelectron spectroscopy studies of nanoparticles dispersed in static liquid. Langmuir 34(33):9606-9616

Pal $S$ et al (2017) Silver-functionalized bacterial cellulose as antibacterial membrane for wound-healing applications. ACS Omega 2(7):3632-3639

Paladini F et al (2013) Effect of silver nanocoatings on catheters for haemodialysis in terms of cell viability, proliferation, morphology and antibacterial activity. J Mater Sci Mater Med 24(4):1105-1112

Panda BP, Patnaik S, Maharana RL (2017) Current trends in design and development of nanopharmaceutical dosage forms. Bioequiv Bioavail Int J 1(1):000104

Patra S et al (2015) Green synthesis, characterization of gold and silver nanoparticles and their potential application for cancer therapeutics. Mater Sci Eng C 53:298-309

Patra JK et al (2018) Nano based drug delivery systems: recent developments and future prospects. J Nanobiotechnol 16(1):1-33

Pinto RJB et al (2013) Antibacterial activity of nanocomposites of copper and cellulose. BioMed Res Int 2013:1-6

Pithawala NA, Jain BK (2021) Physicochemical assessment of AgNPs invigorated antidermatophytic cream from leaves of Crescentia cujete L. A nanopharmaceutical ploy. J Adv Sci Res 12(1 Suppl 2):137-142

Pourzahedi L, Eckelman MJ (2015) Comparative life cycle assessment of silver nanoparticle synthesis routes. Environ Sci Nano 2(4):361-369

Prakash $V$ et al (2017) Analysis of nucleation and growth parameter of silver nanoparticles for sensors. In: 2017 1st International conference on electronics, materials engineering and nano-technology (IEMENTech). IEEE

Prasher P, Singh M, Mudila H (2018) Oligodynamic effect of silver nanoparticles: a review. BioNanoScience 8(4):951-962

Prusty A, Parida P (2014) Development and evaluation of gel incorporated with biogenically synthesized silver nanoparticles. J Appl BioL Pharm 3:1-6

Ramanathan S et al (2018) Eco-friendly synthesis of Solanum trilobatum extract-capped silver nanoparticles is compatible with good antimicrobial activities. J Mol Struct 1160:80-91

Sahana R et al (2014) Formulation of bactericidal cold cream against clinical pathogens using Cassia auriculata flower extract-synthesized Ag nanoparticles. Green Chem Lett Rev 7(1):64-72

Sahu T, Patel T, Sahu S, Gidwani B (2016) Skin cream as topical drug delivery system: a review. J Pharmaceut Biol Sci 4(5):149

Salleh A et al (2020) The potential of silver nanoparticles for antiviral and antibacterial applications: a mechanism of action. Nanomaterials 10(8):1566

Sánchez M et al (2020) Pharmacological update properties of Aloe vera and its major active constituents. Molecules 25(6):1324

Sharma SK, Sharma R (2016) Essential oil constituents and antimicrobial potency of Thuja orientalis grown in Rajasthan. Int J Environ Sci Technol 2(2): $1-7$

Sharma K, Mittal A, Chauhan N (2015) Aloevera as penetration enhancer. Int J Drug Dev Res 7(1):31-43 
Sharma S, Bhatia A, Gakkhar N (2019) Nanotechnology in cancer therapy: An overview and perspectives. Int J Pharmaceut Chem Anal 6(4):110-114

Sharma K (2016) Skin permeation of Candesartan cilexetil from transdermal patch containing Aloe vera gel as penetration enhancer. Asian J Pharm (AJP) 10(2):124-131

Slepička P et al (2020) Methods of gold and silver nanoparticles preparation. Materials 13(1):1

Sonia S, Ruckmani K, Sivakumar M (2017) Antimicrobial and antioxidant potentials of biosynthesized colloidal zinc oxide nanoparticles for a fortified cold cream formulation: a potent nanocosmeceutical application. Mater Sci Eng C 79:581-589

Srivastava P et al (2012) Biological properties of Thuja orientalis Linn. Adv Life Sci 2(2):17-20

Tippayawat P et al (2016) Green synthesis of silver nanoparticles in Aloe vera plant extract prepared by a hydrothermal method and their synergistic antibacterial activity. PeerJ 4:2589

Tutaj K, Szlazak R, Szalapata K et al (2016) Amphotericin B-silver hybrid nanoparticles: synthesis, properties and antifungal activity. Nanomedicine 12:1095-1103
Van de Voorde K et al (2005) Long term use of silver containing nose-drops resulting in systemic argyria. Acta Clin Belg 60(1):33-35

Vega-Villa KR et al (2008) Clinical toxicities of nanocarrier systems. Adv Drug Deliv Rev 60(8):929-938

Vélez E et al (2018) Silver nanoparticles obtained by aqueous or ethanolic Aloe vera extracts: an assessment of the antibacterial activity and mercury removal capability. J Nanomater 2018:1-7

Verma P, Maheshwari SK (2019) Applications of silver nanoparticles in diverse sectors. Int J Nano Dimens 10(1):18-36

\section{Publisher's Note}

Springer Nature remains neutral with regard to jurisdictional claims in published maps and institutional affiliations.

\section{Submit your manuscript to a SpringerOpen ${ }^{\circ}$ journal and benefit from:}

- Convenient online submission

- Rigorous peer review

- Open access: articles freely available online

- High visibility within the field

- Retaining the copyright to your article

Submit your next manuscript at $\boldsymbol{\nabla}$ springeropen.com 